(C) 2015 IEEE. Personal use of this material is permitted. Permission from IEEE must be obtained for all other uses, in any current or future media, including reprinting/republishing this material for advertising or promotional purposes, creating new collective works, for resale or redistribution to servers or lists, or reuse of any copyrighted component of this work in other works. 


\title{
Upper Limb Strength Estimation of Physically Impaired Persons using a Musculoskeletal Model: A Sensitivity Analysis
}

\author{
Marc G. Carmichael, Dikai Liu
}

\begin{abstract}
Sensitivity of upper limb strength calculated from a musculoskeletal model was analyzed, with focus on how the sensitivity is affected when the model is adapted to represent a person with physical impairment. Sensitivity was calculated with respect to four muscle-tendon parameters: muscle peak isometric force, muscle optimal length, muscle pennation, and tendon slack length. Results obtained from a musculoskeletal model of average strength showed highest sensitivity to tendon slack length, followed by muscle optimal length and peak isometric force, which is consistent with existing studies. Muscle pennation angle was relatively insensitive.

The analysis was repeated after adapting the musculoskeletal model to represent persons with varying severities of physical impairment. Results showed that utilizing the weakened model significantly increased the sensitivity of the calculated strength at the hand, with parameters previously insensitive becoming highly sensitive. This increased sensitivity presents a significant challenge in applications utilizing musculoskeletal models to represent impaired individuals.
\end{abstract}

\section{INTRODUCTION}

Musculoskeletal models have been successfully utilized in a range of scientific and clinical applications [1], [2]. It is acknowledged that parameter inaccuracy will affect outcomes generated from the model, and hence the significance of such modelling errors needs to be considered in the context of the desired application. Studies in the literature have identified musculoskeletal model parameters for which particular model-generated outcomes are highly sensitive. For example it has been shown that the forces and torques produced by muscle-tendon actuation are highly sensitive to tendon slack length [3], [4], [5]. Despite the existing studies there are still questions regarding musculoskeletal model sensitivity yet to be addressed. A desired application for musculoskeletal models is the development of individualised surgery plans or providing targeted therapies for patients [1], [2], [6]. Applications such as these require that musculoskeletal models be calibrated so as to represent the physiology and capabilities of individual patients [2]. However it is not yet understood how adapting a musculoskeletal model to represent a subject with impairment will alter its sensitivity characteristics. We hypothesize that musculoskeletal models adapted to represent weaker persons may have an increased susceptibility to parameter inaccuracy, leading to significant implications in their usage.

In this study we investigate the sensitivity of upper limb strength calculated using a musculoskeletal model. Strength

M. G. Carmichael and D. Liu are with the Centre for Autonomous Systems, University of Technology, Sydney, Australia. E-mail: marc.carmichael@uts.edu.au

This work was supported by the Australian Research Council (ARC) Linkage Grant (LP140100950), and Burwell Technologies Pty Ltd. at the hand is calculated using an optimization routine previously developed [6], [7]. Methods currently existing in the literature are then used to quantify how the calculated strength is affected by perturbations in parameters of the model. Muscle-tendon (MT) parameters commonly used in Hill-type muscle-tendon models are investigated as they significantly influence the force capability of the muscles, and their values are difficult to obtain accurately and directly. The sensitivity is first calculated using a musculoskeletal model representing an average healthy male, referred to as the normative model. The analysis is then repeated using duplicate models which are adapted to represent varying degrees of physical impairment, referred to as weakened models. Comparing results between normative and weakened models provides insight into how the modelled impairment affects the sensitivity of the calculated strength.

\section{Methods}

\section{A. Calculation of strength at the hand}

With the model set to a predefined pose, an external force is applied to the hand in a specified direction as shown in Fig. 1. Using a previously developed optimization routine [6], [7] the strength at the hand is calculated as the maximum magnitude of this external force the musculoskeletal system can statically support without any muscles exceeding their force capacities.

In this analysis the strength is calculated in 14 different directions at the hand. Imagining a cube positioned at the centre of the hand, 6 directions correspond to the normals of each face, and 8 point from the cube's centre to its corners. Strength calculations are repeated with the hand at a number of locations distributed throughout the region shown

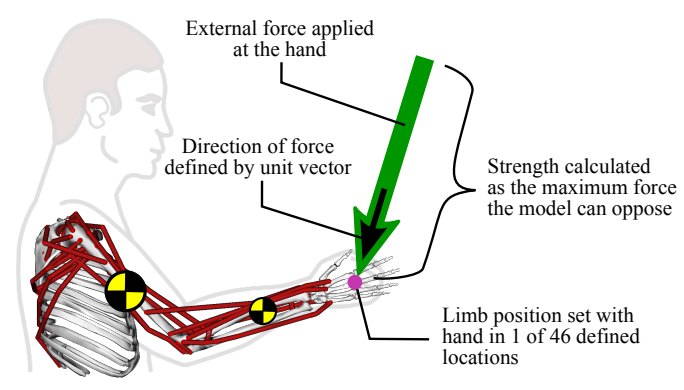

Fig. 1. During strength calculations the upper limb is positioned with the hand at 1 of 46 predefined locations in the workspace. An external force is applied to the hand in 1 of 14 predefined directions. An optimization model [6] calculates the strength at the hand by finding the maximum magnitude of the external force the musculoskeletal model can oppose without exceeding its muscular strength capacity. 


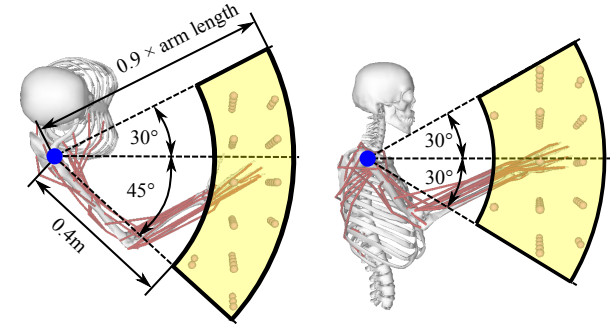

Fig. 2. The region used to define the 46 hand locations where upper limb strength is analyzed. Distance from the hand to the shoulder joint must be greater than $0.4 \mathrm{~m}$ and less than 0.9 times the stretched arm length. The hand must also lay in an area bounded by planes passing through the shoulder. These planes are angled $\pm 30^{\circ}$ from the transverse plane; and $30^{\circ}$ medial $/ 45^{\circ}$ lateral of the sagittal plane.

in Fig. 2. This region was chosen as it contains locations in the frontal workspace commonly reached during everyday activities, and inverse kinematic solutions for the musculoskeletal model are known to exist. A cartesian grid with $10 \mathrm{~cm}$ spacing produced 46 distinct hand locations within this region where strength at the hand is to be analyzed. Kinematic redundancy is resolved by positioning the elbow using the method described in [8] to produce a position consistent with natural human reaching.

\section{B. Upper limb musculoskeletal model}

A musculoskeletal model [9] from the literature is used to calculate upper limb strength. This model has been shown to depict several upper limb characteristics including joint strength [9], muscle moment-arms [10] and stiffness at the hand [11]. In this work the joints of the wrist and fingers are locked, leaving 3 degrees of freedom in the shoulder and 1 degree of freedom in the elbow. Muscles in the model are represented as Hill-type muscle-tendon (MT) actuators, each containing a number of intrinsic parameters [12]. Sensitivity of the upper limb strength is calculated with respect to four of these MT parameters: muscle peak isometric force $\left(F_{m}^{0}\right)$, muscle optimal length $\left(L_{m}^{0}\right)$, muscle pennation at optimal length $\left(\alpha^{0}\right)$, and tendon slack length $\left(L_{t}^{0}\right)$. Remaining MT parameters relating to muscle dynamics are not analyzed as calculation of the strength assumes static conditions, and hence do not affect the strength result.

\section{Strength sensitivity calculation}

To quantify the sensitivity of the calculated upper limb strength we use a perturbation method commonly utilized in existing sensitivity studies [3], [4], [5]. At hand location $i=\{1 \ldots 46\}$ the strength at the hand in direction $j=$ $\{1 \ldots 14\}$ is calculated as $S_{i j}^{0}$ using the method described in [6]. A positive perturbation is then made to the MT parameter being studied $\left(F_{m}^{0}, L_{m}^{0}, \alpha^{0}\right.$ or $\left.L_{t}^{0}\right)$, changing its value from $p^{0}$ to $p^{+}$, and the strength at the hand recalculated as $S_{i j}^{+}$. Similarly, a negative perturbation is made to the same MT parameter changing its value from $p^{0}$ to $p^{-}$, and the strength recalculated as $S_{i j}^{-}$. The sensitivity, represented as $\varepsilon_{i j}$, is then quantified as the normalized change in strength divided by the normalized perturbation of the selected MT parameter (1). Perturbation sizes used in the literature are widely varied, from as small as $\pm 2.5 \%$ [4] up to $\pm 50 \%$ [3] of the parameter's value. A perturbation of $\pm 1 \%$ was chosen so as to achieve a result approximating a partial derivative. For comparison the analysis was repeated using $\mathrm{a} \pm 5 \%$ perturbation and produced nearly identical results.

$$
\varepsilon_{i j}=\left|\frac{\left(S_{i j}^{+}-S_{i j}^{-}\right) / S_{i j}^{0}}{\left(p^{+}-p^{-}\right) / p^{0}}\right|
$$

With respect to a specific MT parameter in a specific muscle group, the sensitivity of the calculated strength is taken as the maximum sensitivity calculated across all 46 hand locations in all 14 directions at the hand (2). This procedure is repeated with each of the four MT parameters being perturbed in each of the 37 muscle groups in the upper limb model

$$
\left.\varepsilon=\max \left(\varepsilon_{i j} \mid \begin{array}{l}
i \in\{1 \ldots 14\} \\
j \in\{1 \ldots 46
\end{array}\right\}\right)
$$

\section{Analysis of modelled physical impairment}

It is hypothesized that upper limb strength calculations will have different sensitivity characteristics when the musculoskeletal model is adapted to represent a subject with physical impairment. This is investigated by repeating the sensitivity analysis with the musculoskeletal model modified to represent persons with varying degrees of physical impairment. The model is made weaker by scaling the peak isometric force parameter $\left(F_{m}^{0}\right)$ of all muscles in the model to $75 \%, 50 \%$ and $37.5 \%$ of their original values. The sensitivity analysis is repeated for each weakened upper limb model, allowing the effect of using weaker models on the sensitivity of the calculated upper limb strength to be observed.

\section{RESUlTS}

\section{A. Sensitivity of calculated strength using normative model}

Fig. 3 shows the sensitivity of the upper limb strength calculated for the normative model (i.e. $100 \%$ of its original $F_{m}^{0}$ values). Each graph corresponds to the MT parameter that was perturbed, with each bar corresponding to the muscle in which this perturbation was applied. Results are limited to the 8 most sensitive muscle groups for each perturbed parameter.

Comparison of the graphs indicate that strength was most sensitive to tendon slack length $L_{t}^{0}$ (Fig. 3a) which produced a sensitivity of 5.18 in the lateral deltoid (DELT2). The anterior deltoid (DELT1) was almost as sensitive with a calculated sensitivity of 4.69. The second most sensitive MT parameter was the muscle optimal length $L_{m}^{0}$ (Fig. 3b), which was also most sensitive in the the DELT2 and DELT1 muscle groups. This agrees with previous studies that have shown that muscle-tendon actuator models have significant sensitivity to $L_{t}^{0}$ and $L_{m}^{0}$ parameters [3].

Less sensitive than both $L_{t}^{0}$ and $L_{m}^{0}$ was the muscle peak isometric force $F_{m}^{0}$ (Fig. 3c). This is somewhat explained by the fact that this parameter scales the force output of the active and passive elements in the muscle-tendon actuator 


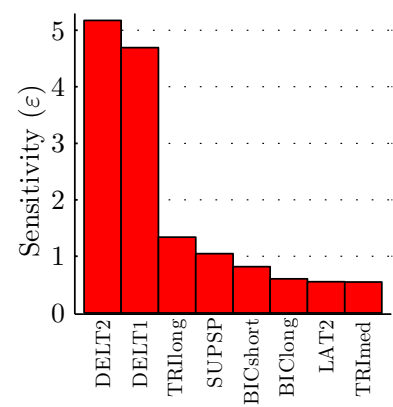

(a) Perturbed parameter: $L_{t}^{0}$

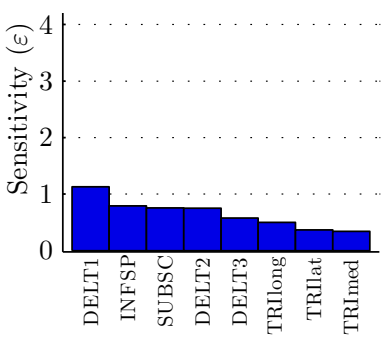

(c) Perturbed parameter: $F_{m}^{0}$

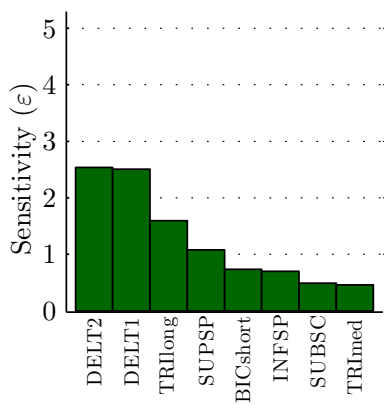

(b) Perturbed parameter: $L_{m}^{0}$

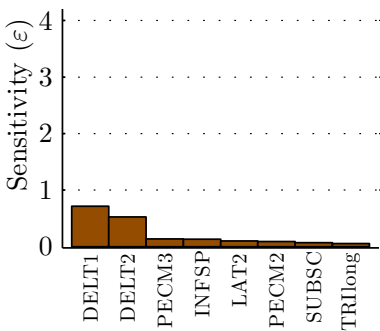

(d) Perturbed parameter: $\alpha^{0}$

Fig. 3. Each subplot shows the 8 most sensitive muscle groups with respect to perturbations made in the following MT parameters: (a) tendon slack length $L_{t}^{0}$, (b) muscle optimal fiber length $L_{m}^{0}$, (c) peak muscle isometric force $F_{m}^{0}$, (d) muscle pennation at optimal length $\alpha^{0}$.

models. If the tendon is assumed rigid, then perturbations in a single $F_{m}^{0}$ parameter are expected to have at most an equally sized effect on the strength (i.e. $\varepsilon \leq 1$ ). All sensitivities calculated with respect to $F_{m}^{0}$ were less than 1, except for DELT1 calculated at 1.13. We attribute this to the nonlinearities resulting from tendon stretch in the model.

The pennation angle $\alpha^{0}$ (Fig. 3d) was relatively insensitive, the largest sensitivity being 0.71 in the DELT1. This is consistent with the findings of others which also found muscle pennation angle to be insensitive [13], [5]. It is noted that several muscles are defined having $\alpha^{0}=0$, and hence a $\%$ perturbation will have no effect on the calculated strength.

\section{B. Sensitivity with weakened models}

Fig. 4 shows the sensitivity calculated with the musculoskeletal model having varying degrees of impairment applied. Similar to the previous figure, each graph corresponds to the MT parameter that was perturbed. Bars are shown in groups of four with each group corresponding to the muscle the parameter was perturbed in. Each individual bar in the groups show the sensitivity results calculated at the four different levels of model weakness. Graphs are limited to showing the 5 most sensitive muscles calculated using the weakest model (37.5\% normative strength).

The results show that the sensitivity of the calculated strength increases as the weakness of the model is made more severe. This was observed across almost all the muscle groups shown. The exception is for the perturbed pennation angle in the PECM3 muscle group, however this had little sensitivity to begin with compared to the other MT parameters.

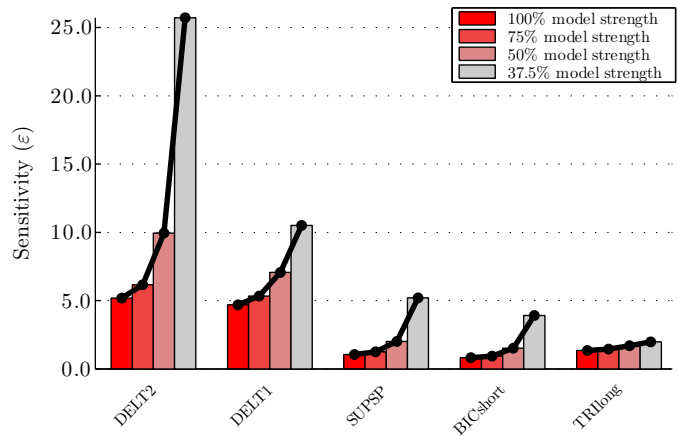

(a) Perturbed parameter: $L_{t}^{0}$

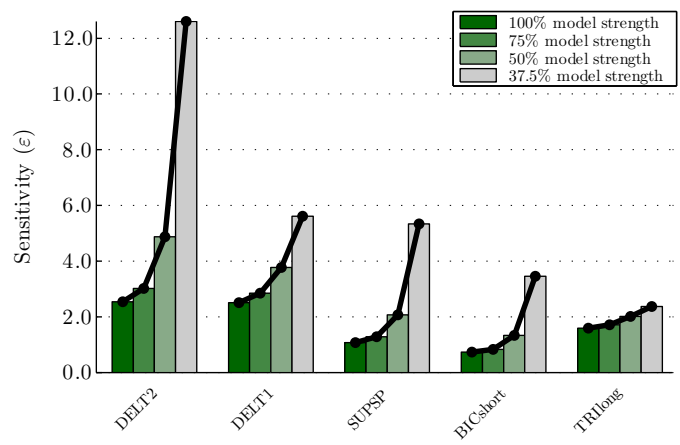

(b) Perturbed parameter: $L_{m}^{0}$

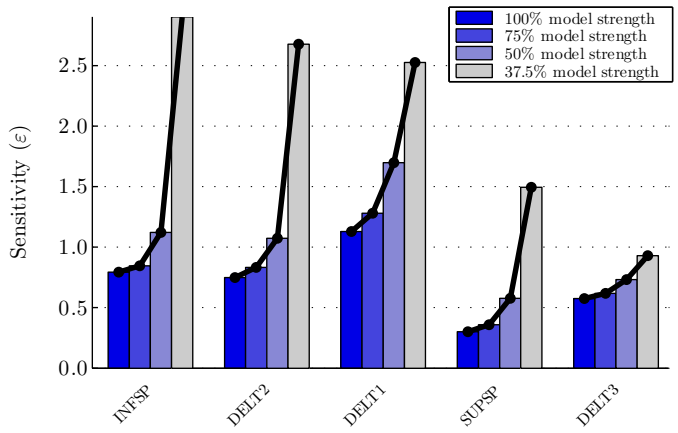

(c) Perturbed parameter: $F_{m}^{0}$

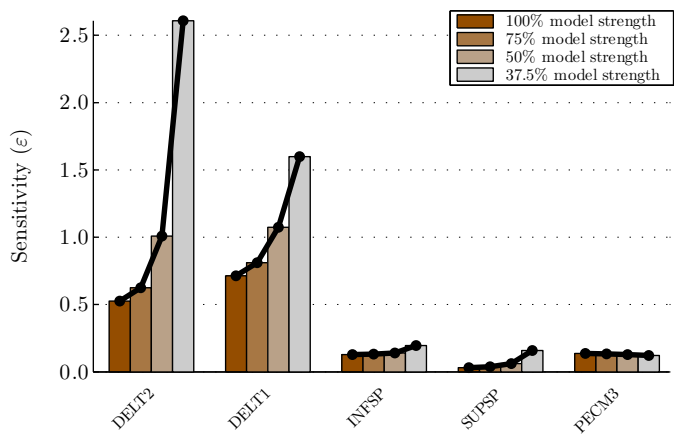

(d) Perturbed parameter: $\alpha^{0}$

Fig. 4. Sensitivity of the strength at the hand, calculated with the upper limb model having various degrees of impairment. The model is made weaker by scaling the peak isometric force $\left(F_{0}^{m}\right)$ parameters in all muscles to $100 \%$, $75 \%, 50 \%$ and $37.5 \%$ of their normative values. The thick black lines added to the top of each group of bars show how the sensitivity increases when a weaker model is used. 


\section{DISCUSSION}

Although it was expected that the calculated strength $\left(S_{i j}^{0}\right)$ would decrease for musculoskeletal models that are adapted to represent persons with physical impairment, the relationship with respect to its sensitivity was unknown. Ideally the strength would have a constant sensitivity, or better yet become less sensitive as modelled impairments are made more severe. However as the musculoskeletal model was weakened from $100 \%$ down to $37.5 \%$ of its normative muscular force-producing capacity, the sensitivity of the calculated strength at the hand grew in an increasing manner. Even parameters which were insensitive with the normative model (e.g. the muscle pennation angle) became highly sensitive when the weakest model was used. This is a significant but undesired result for applications utilizing musculoskeletal models to estimate the strength of persons with physical impairments. For applications such as these, obtaining accurate model parameters is even more essential in order to have confidence in the strength estimations produced.

Given the desire to use musculoskeletal models representing patients with physical impairments in applications such as rehabilitation, solutions that mitigate the increased model sensitivity to parameter errors need to be investigated. The obvious solution is to ensure that accurate parameter values are used in the first place. This has been recognized as a key challenge in the use of musculoskeletal models for individualized modelling in clinical applications. Proposed solutions to this are to use a variety of methods to obtain as accurate parameter values as possible from subject measurements in vivo, including motion capture, force and torque measurements, electromyography and medical imaging [2]. A caveat is that musculoskeletal properties are known to change over time, for example with age and usage [14], [15]. A once-off calibration of models may not be enough, particularly in applications such as rehabilitation where the physiological properties of the patient will vary (hopefully improving) over a relatively short period of time.

It is anticipated that other musculoskeletal models, of either the upper limb and other body segments, will have their sensitivity affected when modelling physical impairments, and hence further work is required. Additionally, it is not clear the extent to which the specific method used to model physical impairment (in this work reducing $F_{0}^{m}$ ) contributes to the change in the model's sensitivity. Alternative methods may allow the development of models less sensitive to its parameters, yet capable of accurately representing an individual and their physical impairments.

\section{CONCLUSION}

In this work the sensitivity of upper limb strength calculated using a musculoskeletal model was analyzed with respect to the model's muscle-tendon (MT) parameters. It was shown that sensitivity varied greatly across the different MT parameters and muscle groups. Results were consistent with existing sensitivity studies with strength being most sensitive to tendon slack length, followed by the muscle optimal fiber length. Muscle peak isometric force was moderately sensitive, and pennation angle showed low sensitivity.

It was observed that the sensitivity of the calculated strength was significantly affected when adapting the model to represent a person with physical impairment. As weaker models were used the sensitivity increased, with parameters that were previously insensitive (e.g. muscle pennation angle) becoming highly sensitive. This result has significant implications on the use of musculoskeletal models for predicting the upper limb strength of persons with physical impairments.

\section{REFERENCES}

[1] P. Favre, J. G. Snedeker, and C. Gerber, "Numerical modelling of the shoulder for clinical applications," Philosophical Transactions of the Royal Society A: Mathematical,Physical and Engineering Sciences, vol. 367 , no. 1895 , pp. 2095-2118, 2009.

[2] B. J. Fregly, M. L. Boninger, and D. J. Reinkensmeyer, "Personalized neuromusculoskeletal modeling to improve treatment of mobility impairments: a perspective from european research sites.," J Neuroeng Rehabil, vol. 9, p. 18, 2012

[3] C. Y. Scovil and J. L. Ronsky, "Sensitivity of a Hill-based muscle model to perturbations in model parameters.," J Biomech, vol. 39, no. 11 , pp. 2055-2063, 2006.

[4] C. Redl, M. Gfoehler, and M. G. Pandy, "Sensitivity of muscle force estimates to variations in muscle-tendon properties.," Hum Mov Sci, vol. 26, pp. 306-319, Apr 2007.

[5] F. De Groote, A. Van Campen, I. Jonkers, and J. De Schutter, "Sensitivity of dynamic simulations of gait and dynamometer experiments to Hill muscle model parameters of knee flexors and extensors.," $J$ Biomech, vol. 43, pp. 1876-1883, Jul 2010.

[6] M. Carmichael and D. Liu, "Estimating physical assistance need using a musculoskeletal model," Biomedical Engineering, IEEE Transactions on, vol. 60, no. 7, pp. 1912-1919, 2013.

[7] M. G. Carmichael and D. Liu, "Towards using musculoskeletal models for intelligent control of physically assistive robots," in Engineering in Medicine and Biology Society,EMBC, 2011 Annual International Conference of the IEEE, pp. 8162 -8165, Sept 2011.

[8] H. Kim, L. Miller, A. Al-Refai, M. Brand, and J. Rosen, "Redundancy resolution of a human arm for controlling a seven DOF wearable robotic system," in Engineering in Medicine and Biology Society,EMBC, 2011 Annual International Conference of the IEEE, pp. $3471-3474$, Aug 2011.

[9] K. R. S. Holzbaur, W. M. Murray, and S. L. Delp, "A model of the upper extremity for simulating musculoskeletal surgery and analyzing neuromuscular control," Annals of Biomedical Engineering, vol. 33, pp. 829-840, June 2005.

[10] C. J. Gatti, C. R. Dickerson, E. K. Chadwick, A. G. Mell, and R. E. Hughes, "Comparison of model-predicted and measured moment arms for the rotator cuff muscles," Clinical Biomechanics, vol. 22, no. 6, pp. $639-644,2007$.

[11] X. Hu, W. M. Murray, and E. J. Perreault, "Muscle short-range stiffness can be used to estimate the endpoint stiffness of the human arm," Journal of Neurophysiology, vol. 105, no. 4, pp. 1633-1641, 2011

[12] L. Schutte, M. Rodgers, F. Zajac, and R. Glaser, "Improving the efficacy of electrical stimulation-induced leg cycle ergometry: an analysis based on a dynamic musculoskeletal model," Rehabilitation Engineering, IEEE Transactions on, vol. 1, pp. 109-125, Jun 1993.

[13] F. E. Zajac, "Muscle and tendon: properties, models, scaling, and application to biomechanics and motor control," Critical reviews in biomedical engineering, vol. 17, no. 4, pp. 359-411, 1989.

[14] R. A. Brand, D. R. Pedersen, and J. A. Friederich, "The sensitivity of muscle force predictions to changes in physiologic cross-sectional area.," J Biomech, vol. 19, no. 8, pp. 589-596, 1986.

[15] J. A. Friederich and R. A. Brand, "Muscle fiber architecture in the human lower limb.," J Biomech, vol. 23, no. 1, pp. 91-95, 1990. 